\title{
Guidelines for Exfoliation, Characterisation and Processing of Layered Materials Produced by Liquid Exfoliation
}

Claudia Backes, ${ }^{1,2, \sharp}$ Thomas M. Higgins, ${ }^{1,2, \ddagger}$ Adam Kelly, ${ }^{1}$ Conor Boland, ${ }^{1}$ Andrew Harvey, ${ }^{1}$ Damien Hanlon ${ }^{1}$ and Jonathan N. Coleman ${ }^{1, *}$

${ }^{1}$ School of Physics and CRANN \& AMBER Research Centers, Trinity College Dublin, Dublin 2, Ireland

${ }^{2}$ Chair of Applied Physical Chemistry, Ruprecht-Karls Universität Heidelberg, Im Neuenheimer Feld 253, 69120 Heidelberg, Germany

$\$$ These authors contributed equally

*colemaj@tcd.ie

\begin{abstract}
Liquid phase exfoliation has become an important method for the production of large quantities of 2-dimensional nanosheets. This method is versatile, having been used to produce dozens of different 2D materials in a range of stabilizing liquids. The resultant liquidsuspended nanosheets have been characterized in great detail and have been processed into a number of structures for a wide range of applications. This has led to a growing number of researchers adopting this method. As a result, best practise in terms of experimental procedure has evolved rapidly over recent years. As experimental complexity has increased it has become more and more difficult to discuss the rational behind a chosen experimental procedure in full detail using standard "Methods" sections due to the frequent use of procedures developed in related prior reports. This can make it difficult to reproduce complex procedures and acts as a barrier to new researchers entering the field. To address this shortcoming, here we describe in detail the experimental methods and best practice used in our group when producing liquid exfoliated nanosheets.
\end{abstract}




\section{Introduction}

Over the last decade the study of 2-dimensional (2D) materials has evolved into one of the hottest areas of nano-materials science. ${ }^{1-3}$ Although $2 \mathrm{D}$ oxides ${ }^{4}$ and clays ${ }^{5}$ have been studied for some time, the rapid growth of this area was driven by research into graphene, ${ }^{6}$ a $2 \mathrm{D}$ material with superlative properties and unprecedented applications potential. As research into graphene deepened, the field simultaneously began to broaden out to encompass other 2D materials starting with boron nitride $(\mathrm{BN})$ and molybdenum disulphide $\left(\mathrm{MoS}_{2}\right)$, eventually progressing on a broad front including exotic structures such as silicene and black phosphorous. This expansion has been driven by the fact that, like graphene, many 2D materials have very exciting physical and chemical attributes. ${ }^{1,3}$

In order to study or utilize 2D materials, control of their production is critically important. In the early days 2D nanosheets were produced by mechanical exfoliation. ${ }^{7}$ However, as the field progressed, the need for more scalable techniques became apparent. Very crudely, we can divide modern production techniques into two classes: those such as chemical vapour deposition $^{8}$ and thermally assisted conversion ${ }^{9}$ which produce high quality, extended monolayer films on substrates and those that transform layered crystals into large quantities of small nanosheets, usually in liquids. Here we will focus on the latter class, usually referred to as liquid exfoliation methodologies. ${ }^{2}$

In general, these methods result in 2D nanosheets with lateral sizes in the range $100 \mathrm{~nm}-100$ $\mu \mathrm{m}$ and thicknesses ranging from 1-10 monolayers. These nanosheets can be produced in a number of liquids at a range of concentrations. Depending on the production method, they can be extremely defective or almost defect-free. A number of such techniques are widely used; for example, reduced graphene oxide is produced by chemical oxidation of graphite followed by reduction. ${ }^{10}$ Alternatively, graphene can be produced by electrochemical exfoliation of graphite in appropriate electrolytes. ${ }^{11}$ A number of layered materials, including graphite and $\mathrm{MoS}_{2}$ can be exfoliated by ion intercalation while layered oxides and hydroxides are generally exfoliated by ion exchange. ${ }^{4}, 12$ These methods have many advantages such as low cost or high monolayer selectivity but also disadvantages such as complexity or use of toxic chemicals. In particular, all are used in niches to exfoliate a small group of layered materials.

However, there is one exfoliation technique, generally termed liquid phase exfoliation (LPE), which is more versatile than most, having been applied to a wide range of layered materials. ${ }^{13}$, ${ }^{14}$ This method involves the production of few-layer nanosheets by applying high shear ${ }^{15}$ or 
ultrasound $^{13}$ to layered crystals in certain stabilizing liquids (i.e. appropriate solvents ${ }^{16}$ and surfactant ${ }^{17}$ or polymer solutions ${ }^{18}$ ). In each case, interactions at the liquid - nanosheet interface reduce the net exfoliation energy and stabilize the nanosheets against aggregation. ${ }^{19}$ The resultant dispersions are quite stable and can be produced at concentrations ${ }^{20}$ exceeding 1 $\mathrm{gL}^{-1}$. LPE has been applied to a wide range of $2 \mathrm{D}$ materials including graphene, ${ }^{14,21,22} \mathrm{BN},{ }^{23}$ transition metal dichalcogenides (TMDs), ${ }^{13,}{ }^{24}$ transition metal oxides (TMOs), ${ }^{25}, 26 \mathrm{GaS},{ }^{27}$ phosphorene, ${ }^{28,}{ }^{29} \mathrm{Ni}(\mathrm{OH}) 2^{30}$ and MXenes. ${ }^{31}$ This method has a very important advantage that it is simple and potentially scalable and cheap. ${ }^{15,} 32$ It should be noted that this method produces mostly few-layer nanosheets (typically $\sim 1-10$ stacked monolayers), with individualized monolayer contents which are low compared to the methods described above. In addition, the lateral size distribution of the nanosheets produced by LPE can be broad, e.g. 40-400 $\mathrm{nm}$ for $\mathrm{MoS}_{2}{ }^{33}$ The mean of the size distribution tends to vary from material to material with $\mathrm{MoS}_{2}$ and $\mathrm{WS}_{2}$ giving nanosheets which are considerably smaller than those of graphene and black phosphorous. However, we note that a range of methods have been demonstrated to select nanosheets by size and enrich the monolayer population. ${ }^{33,} 34$ The resultant dispersions can very easily be processed into nanostructured materials by a range of methods such as spray deposition, ${ }^{35}$ inkjet printing, ${ }^{36,37}$ and freeze drying. ${ }^{38}$ These structures have been used in a wide range of applications from battery electrodes ${ }^{39}$ to barrier composites $^{40,41}$ to photodetectors. ${ }^{42,43}$

In its simplest form, LPE can be achieved with a kitchen blender and household soap. ${ }^{44}$ As such, liquid exfoliation appears to be a straightforward and low-cost process. However, many parameters need to be carefully controlled, making reproducibility a challenging task. Very often the outcome depends on subtleties in the process which are often not discussed to the level of detail that would be desired for other experimentalists to reproduce the results. This is especially because in most cases only successful experiments are reported - often giving little room to discuss the trial and error along the way and hence the influence of certain parameters on the outcome.

Here we have attempted address this with an illustration and discussion of the state of the art methods and protocols developed in our lab to perform liquid exfoliation, size selection, basic spectroscopic and microscopic characterization, as well as further processing into functional films. We hope this article will provide valuable guidance to other researchers in the area, help to improve reproducibility and, in general, assist with the continued rapid development of this exciting field. 


\section{Discussion of Methods}

\section{Liquid exfoliation and size selection}

In general, liquid exfoliation can be considered as a three step process. Firstly, the weak interlayer attractions between adjacent sheets need to be overcome by imparting energy during the actual exfoliation process. Secondly, the nanosheets need to be stabilized against reaggregation by suitable solvents or surfactants. They play a dual role as they minimise the net energy cost of the exfoliation while also adsorbing to the nanosheet surface, shielding them from restacking in the liquid. Thirdly, size-selection is often required. This is because the as-produced dispersions are highly polydisperse (figure 1B), containing nanosheets with a range of sizes and thicknesses (figure 1C). Equally serious, such dispersions typically display low monolayer contents. As such, for many of the application areas where liquid exfoliated nanosheets are well suited (such as printed optoelectronic and electrochemical devices), optimized performance is very unlikely to arise through direct use of the native dispersions. This is especially true for those applications requiring the direct bandgap luminescence properties that are conferred only by monolayers. Therefore, to tap the full potential of nanosheet materials in applications it is clear that size selection must be an integral part of the material production process.

Many strategies exist for both exfoliation and size selection, which strongly depend on the starting material and the desired outcome. In this article, we only review methods that are applied or were developed in our lab even though various alternatives are described throughout the literature. The reader is referred to a number of recent review articles on exfoliation in liquid media. ${ }^{2,45-49}$ Thus, we do not intend to give a comprehensive overview of the entire field, but rather wish to share our experience gathered over the past few years with other researchers working in this field. In the first sections, we will address questions such as which medium (e.g. solvent or surfactant) or exfoliation method (e.g. sonication or shear exfoliation) is more suitable and how to efficiently size-selected nanosheets using benchtop centrifuges. For a visualization of the specific protocol we use to make our standard TMD samples, the reader is referred to our video publication..$^{50}$ In the supporting information we provide an overview of the specific consumables and setups that we routinely use.

\section{General exfoliation protocol}

In a typical exfoliation protocol, the bulk material (see supplementary information for examples) is immersed in a suitable solvent, or aqueous surfactant or polymer solution and 
subjected to ultrasonication using a bath or tip sonicator, or shear force mixers, respectively. After the actual exfoliation, the dispersion is then subjected to centrifugation to remove unexfoliated material and/or perform size selection. In the case of sonication, typical processing times are on the order of a few hours, whereas shear exfoliation requires longer processing times to yield the same concentration. However, larger volumes (i.e. litres) can be produced using shear exfoliation, whereas sample volumes are usually less than $500 \mathrm{~mL}$ using sonication (depending on the sonicator). Further advantages and disadvantages of the different exfoliation methods are summarized below.

A number of process parameters are crucial for the outcome of the exfoliation. These include solvent or stabilizer (and its concentration), initial concentration of the bulk material, process time and volume. In addition to these common aspects, other parameters which depend on the specific exfoliation method also need to be considered, such as power output and amplitude of sonicators, or the rotational speed of the blades within shear force mixers. We also find that it is crucial to control the temperature during the exfoliation; over-heating usually results in lower dispersed concentration and can lead to significant chemical degradation of the nanosheets, especially in the case of more exotic inorganic layered materials. Before discussing more specific parameters related to the choice of solvent or exfoliation method, in the following we will first comment on the more general parameters.

Stabilization using additive-free solvents can be described within the framework of solution thermodynamics, which predicts that efficient stabilization or dissolution occurs when the net energetic cost of mixing is minimized. Since nanomaterials are rather large and rigid compared to molecules, the entropic contribution can be neglected which means that stabilization is achieved when the solubility parameters (such as the surface tension or Hansen parameters) of solvent and solute match. ${ }^{19,51-53}$ Typical solvents that are known to give stable dispersions include $\quad N$-methyl-2-pyrrolidone (NMP), $\quad N$-cyclo-2-pyrrolidone, dimethlyformamide (DMF), dimethylsulfoxide (DMSO) and isopropanol (IPA). Alternatively, stabilizers can be added, for example surfactants in an aqueous solution. These adsorb by non-covalent interactions to the nanosheet surface, for example, by their non-polar tail in the case of classical amphiphiles. In this case, the surfactant head group interacts with the liquid environment and prevents the nanosheets from reaggregation by electrostatic and/or steric repulsion. ${ }^{54}$ Popular aqueous surfactants include sodium cholate (SC) or related bile salts, sodium dodecyl benzyl sulfonate (SDBS), sodium dodecyl sulfate (SDS) as anionic surfactants, cetyl trimethyl ammonium bromide (CTAB) as cationic surfactant or Triton X100 
or Brij as non-ionic representatives. We find that stable dispersions can be produced when the surfactant concentration is kept below its critical micelle concentration. Typically, we use SC in water with a concentration of 2-6 $\mathrm{gL}^{-1}$. A comprehensive understanding of the role of surfactant concentration is currently lacking, however we have found that nanosheet size and thickness in a stock dispersion can vary with the surfactant concentration used. ${ }^{44}$ This was demonstrated for $\mathrm{MoS}_{2}$ exfoliated and stabilized in aqueous SC using turbulence-assisted shear exfoliation. While this offers some possibility of in situ control, it also means that the surfactant concentration may have a larger impact on the outcome of the exfoliation than other process parameters.

Irrespective of the exfoliation method, stabilizer and material, we recommend that a precleaning step of the raw material be conducted. This is because we have experienced that the purity of the commercially available materials is often unpredictable, leading to problems regarding the reproducibility in the exfoliated product. Accordingly, the general recommendation is to perform a two-step exfoliation process. In the first step, the material in the medium of choice should be subjected to a comparatively short $(\sim 20 \%$ of the planned duration for the actual exfoliation) exfoliation with the method of choice. After this initial step, the dispersion should be subjected to centrifugation at intermediate centrifugal acceleration ( $5000 \mathrm{~g}$ for inorganic materials) after which the supernatant is to be decanted and discarded and the sediment collected in fresh solvent/surfactant. This can then be followed by a second, longer exfoliation. This precaution allows much of the impurities (and very small nanosheets) to be removed. We have found this to be particularly crucial when working with ionic surfactants, as the impurities are often ionic and therefore, can destabilize the surfactant dispersion via charge screening effects.

Furthermore, we unanimously find that a higher initial concentration of the nanomaterial will also give a higher concentration of exfoliated nanosheets- at least up to a certain point where the dispersed concentration saturates. While the initial concentration that corresponds to the onset of this dispersed material plateau is material and exfoliation method dependent, it is typically $>30 \mathrm{~g} / \mathrm{L} .^{13,15,26,27,30,44,55,56}$ Similarly, longer processing times yield higher dispersed concentrations (but not linearly). ${ }^{13}, 15,26,27,30,44,55-58$ While this can also change the length and thickness distributions in the stock dispersion, ${ }^{57}$ this effect can be balanced by appropriate size-selection techniques. However, it should be emphasized that it is currently not yet clear whether longer process times will introduce defects. 


\section{Choice of medium}

The first question that needs to be considered when designing a set of experiments involving liquid-exfoliated nanosheets pertains to the medium that is used to stabilize the nanosheets. The answer to this question depends strongly on the material and the purpose of its production. It is therefore not a trivial decision to be made. With the aim of providing a rough guide, we will summarise the pros and cons for the main classes of stabilizers from our perspective in the following.

Aqueous surfactant or biomolecule solutions are widely used as stabilizers in LPE. ${ }^{17,46 \text {, }}$ 54, 59, 60 Usually, the exfoliation, stabilization and size selection is quite robust and reproducible giving access to long-term stable, high quality dispersions that are suitable for many applications and fundamental studies. The medium is environmentally friendly and compatible with many processing techniques such as vacuum filtration and spraying (when the surfactant concentration is minimised). However, printing from aqueous surfactant still remains challenging as it is difficult to control the rheological properties. ${ }^{45}$ In addition, there are two other major downsides. Firstly, some materials (such as $\mathrm{MoO}_{3}{ }^{25}$ or black phosphorus ${ }^{55,61}$ ), are chemically unstable in the aqueous environment and degrade readily. Secondly, it is difficult to remove the surfactant from the nanosheet surface completely after processing which can potentially deteriorate the resulting network properties. Strategies to remove the surfactant include minimizing its concentration to begin with, for example, after the size selection (see below) or though dialysis. However, this can cause reaggregation of the nanosheets in the liquid and potentially interfere with further solution processing. Alternatively, the nanosheets can first be deposited and then rinsed with water followed by annealing. Nonetheless, from our experience, a complete removal is often not possible, as surfactant can be trapped between reaggregated nanosheets within the network. In addition, only a few systematic studies exist on the impact of the chemical structure of the surfactant on the degree of exfoliation and especially nanosheet size with little empirical data available apart from the most commonly used surfactants (e.g. SC or sodium dodecyl benzene sulfonate). Overall, we nonetheless recommend the use of aqueous surfactant solution, as long as it is compatible with the material to be exfoliated; from our experience, residual surfactant does not cause major problems in most application areas. Furthermore, aqueous surfactant systems do not require additional health precautions unlike some alternative non-aqueous solvents and are therefore highly convenient for dispersion and film preparation. 
Alternatively, certain solvents can be used to produce colloidally stable dispersions, typically in the absence of additional stabilizers ${ }^{13,14,23}$ even though additives can further enhance stability or degrees of exfoliation. ${ }^{62}$ One main advantage of using solvents is that chemically unstable materials such as black phosphorus can be protected against degradation through a stabilizing solvation shell. ${ }^{55}$ In addition, it is easier to tune rheological properties which is required for certain processing techniques such as inkjet printing ${ }^{36}$ and removal of the solvent is typically easier than for surfactants. On the other hand, many suitable solvents such as $N$-methyl-2-pyrrolidone (NMP) suffer from high boiling points and are often toxic. Another consideration is that certain solvents can degrade and polymerise ${ }^{63}$ which can dramatically alter the properties of the exfoliated material while also hampering subsequent spectroscopic and microscopic characterization. Furthermore, the degree of exfoliation typically tends to be lower than for surfactants under equal production conditions. While solvent blends have been identified as promising alternatives (for example of alcohols or acetone and water $)^{64-67}$ the dispersions in this case can suffer from long-term instability due to evaporation of the more volatile solvent component.

Polymers (or proteins) present the third main class of potentially suitable stabilizers. ${ }^{68-73}$ Their main advantage lies in the ability to stabilize the dispersion in either aqueous or organic environment. However, this field of research is in general rather immature and only rarely used in our laboratory unless polymer composites are the target application. We would also like to note that we find that the degree of exfoliation in this case to be lower than for small molecule stabilizers and that increased viscosities and densities in polymer solutions may require significant alterations to established size selection procedures (see below).

\section{Choice of exfoliation method}

In the following, we would like to discuss our established exfoliation methods such as bath or tip sonication ${ }^{13,17,20,26,27,30,55,57,74}$ or shear exfoliation (Figure 1D-G). ${ }^{15,44,56}$ We note that other techniques such as ball milling ${ }^{75-78}$ or approaches based on exploiting fluid dynamics in a hydrodynamic apparatus ${ }^{79-81}$ can be used as alternative strategies. Although we describe a number of pros and cons of these different exfoliation methods below, again, the choice of the exfoliation method should be material dependent. For example, while graphite is readily exfoliated for all these methods, ${ }^{15}, 44,57,58,74$ this may not be the case for other materials. In particular, for TMDs the quality of exfoliation (as measured by the yield of large thin nanosheets) is considerably better when using tip sonication compared with bath sonication or shear mixing. On the contrary, for GaS, bath sonication is the most effective 
approach. ${ }^{27}$ Therefore, in general, when attempting to exfoliate a new material it is important to not only consider the stabilizer, but also to test various available exfoliation methods.

As has been mentioned, there are two varieties of sonication; one features an ultrasonic tip which is positioned in the dispersion media and the other is called bath sonication, whereby a vial containing the material to be dispersed is itself immersed in a water bath that is perturbed by ultrasonic vibrations (figure $1 \mathrm{D}$ and $\mathrm{E}$ respectively). In the former method, energy is imparted to the dispersion media directly, whereas for the sonic bath method the energy must travel through the tank and dispersion vial before reaching the nanosheets. Although the scalability of sonication to produce few layer nanosheets has not been demonstrated (compared with shear exfoliation and ball milling), they remain the primary means for exfoliation in our laboratory.

For layered inorganic materials, tip sonication is preferred over bath sonication due to the higher production rates, (i.e. more exfoliated material is produced in shorter times, with concentrations in the range of $\sim 1 \mathrm{~g} / \mathrm{L}$ in $<24 \mathrm{~h}$ for initial bulk material concentrations of 30$50 \mathrm{~g} / \mathrm{L}$ ). In this case, various sonic tip configurations are available with different probe sizes, shapes and process controllers. We find that the type of setup has little impact on the material quality that is produced, provided that the probe diameter is adjusted to the volume of the dispersion as suggested by the manufacturers. In addition, the sonic probe should be polished frequently, as it wears down on prolonged sonication. This can affect the dispersion quality. The setup we typically use is shown in figure 1D. It consists of a solid flathead tip with a diameter of $\sim 2 \mathrm{~cm}$ that is immersed into $80 \mathrm{~mL}$ of a dispersion in a metal beaker. To set up the sonication, the sonic tip is lowered into the dispersion to the bottom of the beaker and then lifted up by $1 \mathrm{~cm}$. Sonication amplitudes in this case are typically $60 \%$. We find that lower amplitudes tend result in poorer exfoliation while higher amplitudes to cause damage to the sonicator when operated frequently. Typical sonication times are 5-7 h. From our experience, it is absolutely crucial to prevent heating of the sample. This is not only because heating of the sample can deteriorate the properties of the material, but also because dispersed concentrations are lower. While cooling can be achieved by positioning the metal cup in an ice bath, it is far more ideal to install a chiller system as otherwise the ice must be replenished every 2 hours or so. In addition, keeping the dispersion cool is greatly assisted by the use of pulsing tip through the device controller. We routinely use 6 seconds on and 2 seconds off ratio.

For a given combination of sonicator, operating conditions, cooling, sonication time and material, the outcome of tip sonication appears rather reproducible both in terms of dispersed 
concentration and size distribution. We have found that the probe should be exchanged every 3-5 months when subject to heavy operation. However, please note that many parameters are involved that are currently still poorly understood. As such, deviations from this procedure will likely result in a different quantitative outcome of the exfoliated material.

In general, bath sonication may offer a lower-cost alternative to tip sonication, and additionally, in some cases we found that this approach led to better exfoliation (such as for $\mathrm{GaS}^{27}$ ). The energy input into the sample is also lower, as it is less localised, so that less material fragmentation (breaking of bonds in the nanosheet) can be expected. However, longer processing times are required to achieve an equivalent concentration of dispersed material and, in general, the process is less reproducible. We found that the outcome varies considerably depending on the specific bath used, the filling level of the bath and the positioning of the vial within the bath. Our recommendation is to use small vials and place them in hot spots, as illustrated in figure 1E. While more reproducible results can be obtained by rotating the sample in the bath during the sonication, this will also result in a lower dispersed concentration for an equivalent sonication time.

Alternatively to sonication, we use shear exfoliation in rotor stator mixers (figure $1 \mathrm{~F})^{15}$ or in blenders with rotating blades. ${ }^{44,56}$ Surprisingly, even the kitchen blender displayed in figure $1 \mathrm{G}$ can be used for LPE offering a very low cost alternative to sonication. ${ }^{44}$ Please note that these household kitchen blenders are not designed to operate in organic solvents and can be destroyed when done so. The yield of exfoliated material is not strongly dependent on the processed volume so that shear exfoliation is a scalable process with high production rates. ${ }^{15}$, 44, 56 The quality of the material produced in terms of dispersed concentration and degree of exfoliation is comparable to sonication in the case of graphene, with the added advantage that fragmentation events are limited to the vicinity of the rotor so that nanosheets tend to be larger. However, for layered inorganic materials such as TMDs, this does not seem to be the case and dispersed concentrations are typically in the range of $\mu \mathrm{g} / \mathrm{L}$ in $<24 \mathrm{~h}$. Consequently, at present the majority of our samples are produced using tip or bath sonication.

\section{Size selection}

A major problem with LPE is the limited control over the exfoliation process resulting in polydisperse samples containing broad nanosheet size and thickness distributions. Usually such samples do not fulfil the requirements needed to conduct studies on fundamental properties, or to unravel the application potential in the numerous areas of interest. Hence, post-production size selection is required. We have recently shown that a procedure we term 
liquid cascade centrifugation (LCC) offers an exciting strategy, ${ }^{33}$ in particular, because it is highly versatile and can be carried out using benchtop centrifuges. This is a multi-step procedure whereby various cascades can be designed according to the desired outcome. To demonstrate this process, a general cascade is portrayed in Figure 1H. It involves multiple centrifugation steps whereby each features a higher rotation speed than the last. After each step the sediment is retained and the supernatant is then used in the proceeding stage. As a result, each sediment contains nanosheets in a given size range which have been "trapped" between two centrifugation stages with different speeds. Critical to LCC, the resulting sediment can be redispersed completely by mild sonication in the respective medium, enabling any nanosheet concentration that is desired as well as modification of the concentration of any additives (such as polymers or surfactants). Importantly, virtually no material is wasted in LCC, resulting in the collection of relatively large masses of sizeselected nanosheets. We have applied this procedure to a number of liquid-exfoliated nanosheets including ${ }^{33} \mathrm{MoS}_{2}$ and $\mathrm{WS}_{2}$ as well as $\mathrm{Ni}(\mathrm{OH})_{2},{ }^{30} \mathrm{GaS},{ }^{27}$ black phosphorus ${ }^{55}$ and graphene ${ }^{82}$ in both solvent and surfactant systems.

To achieve efficient size selection, it is critical to remove the supernatant from the sediment as completely as possible as has been illustrated in figure 1I-L. This also means that for this procedure to work, the centrifugation time has to be long enough to allow the majority of the nanosheets to sediment to the bottom of the vial. We note that this does not require centrifugation to equilibrium (opposed to density gradient ultracentrifugation). $59,70,71$ From our experience, we obtain a good separation of supernatant and pellet-like sediment after $2 \mathrm{~h}$ of centrifugation when the filling height of the dispersion in the vial is $<10 \mathrm{~cm}$. Centrifugation times should be extended if greater filling heights are used. The procedure can generally be applied to various materials in various stabilizers. However, depending on the density of the material and density and viscosity of the medium, centrifugal accelerations and/or centrifugation times will require adjustment. In first approximation, nanosheet size selection occurs by nanosheet mass in such a standard cascade making it difficult to select large, thin nanosheets. However, we would like to note that clever design of secondary cascades involving long, low-speed centrifugations to remove thicker material and short, high-speed centrifugations to remove very small nanosheets has shown some potential to overcome this limitation. ${ }^{33}$ 

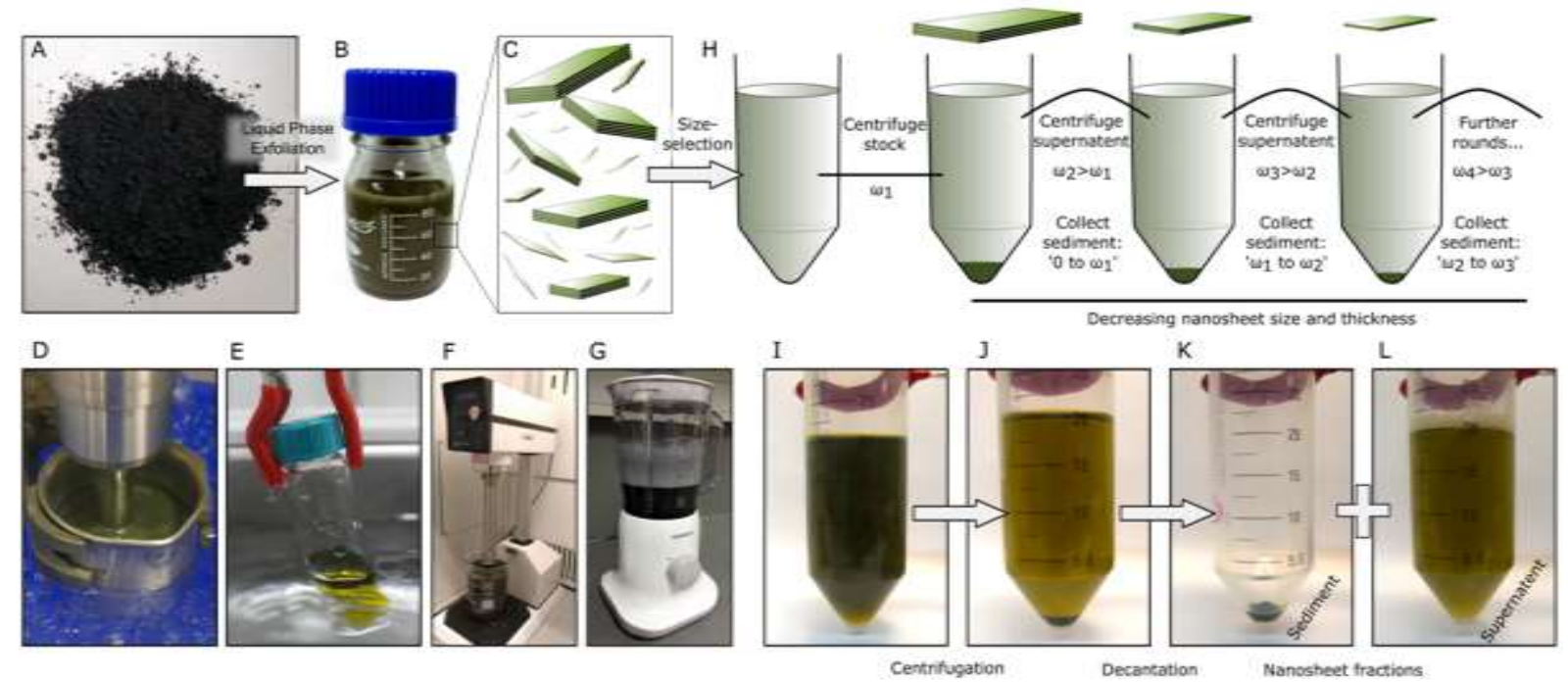

Figure 1: Illustration of liquid exfoliation and size selection. A) Photograph of a typical starting material (here $\mathrm{WS}_{2}$ powder) yielding B) colloidally stable coloured dispersions after the exfoliation containing C) a mixture of nanosheets with varying lateral sizes and thicknesses. Panels D-G) show photographs of setups used for the exfoliation. D) Sonic tip with ice cooling, E) sonic bath with a vial in a hotspot, F) rotor stator mixer, G) household kitchen blender. H) Schematic of the liquid cascade centrifugation with subsequently increasing rotational speeds $\omega$. The supernatant after each step is subjected to another centrifugation at higher centrifugal acceleration. Size-selected nanosheets are collected as sediments. I) Photograph of a centrifugation tube containing an aqueous surfactant dispersion of $\mathrm{WS}_{2}$ before centrifugation. Typical centrifugation times should be 2 hours for filling heights up $10 \mathrm{~cm}$ to allow the heavy material sufficient time to travel to the bottom of the vial. An example of a centrifugation tube after the centrifugation is shown in $\mathrm{J}$ ). To achieve efficient size selection, the supernatant should be removed as completely as possible (by pipetting) resulting in nanosheet fractions as shown in $\mathrm{K}$ and $\mathrm{L}$. A main advantage of this size selection procedure is that the size selected nanosheets in the sediment can be redispersed in reduced volume and therefore at any desired concentration.

\section{Basic characterization}

Regardless of the intended purpose of the produced LPE nanosheets, basic spectroscopic and microscopic characterization is essential. This is particularly important because the LPE and size selection are, at present, rather poorly understood and therefore it is difficult to gauge the outcome of a given LPE and size selection protocol with a given starting material without first conducting extensive experimentation. As such, it is necessary to confirm the outcome of exfoliation and nature of the material that is produced. Below, we recommend a basic set of characterization methods that yield valuable information using equipment that is available to many researchers. This includes techniques such as UV/Visible and Raman spectroscopies, which provide insights regarding structural integrity and very often size/thickness information, as well as microscopic characterization by transmission 
electron microscopy (TEM) and atomic force microscopy (AFM) to confirm shape, morphology, size and thickness of the nanosheets.

\section{Spectroscopic characterization}

The most basic and straight forward characterization for colloidal dispersions is UV/Vis spectroscopy. From our experience, the quantity of information that can be extracted from this simple spectroscopic technique is exceptional and often underestimated. Firstly, UV/Vis transmission spectroscopy can provide information on the structure of the exfoliated nanosheets, as especially inorganic layered materials have well documented excitonic transitions ${ }^{83}$ that are characteristic for the material and even its polytype or phase. ${ }^{63,84,85}$ Secondly, information on the concentration can be extracted. ${ }^{33,34,63,82,86}$ However, for this to be performed reliably, it is essential to be aware that when transmittance mode is used, extinction and not absorbance spectra are obtained. This means that the quantity that is measured contains information related to both the absorbance and the scattering of light due to the nanomaterial. ${ }^{27,} 30,34,55,82,87$ In addition, although it is commonly known that the scattering component of the extinction coefficients is size-dependent, it is also the case that the absorbance coefficient is dependent on material size due to edge and confinement effects. ${ }^{33,34}$ Unfortunately, a detailed discussion is beyond the scope of the manuscript and the reader is referred to the original work related to this subject. ${ }^{33,}{ }^{34} \mathrm{We}$ would nonetheless like to exemplarily show (with $\mathrm{WS}_{2}$ and $\mathrm{MoS}_{2}$ ) that nanosheet size and thickness information is encoded in optical extinction spectra (figure 2A-D). Note that we have made similar observations for other layered materials such as graphene, ${ }^{82} \mathrm{GaS},{ }^{27}$ black phosphorus ${ }^{55}$ and $\mathrm{Ni}(\mathrm{OH})_{2} \cdot{ }^{30}$

Changes in the electronic band structure of layered materials as a result of both edge and confinement effects are reflected in changes in the magnitude and position of the excitonic transitions. Therefore, optical extinction spectra are expected to change as a function of nanosheet lateral size and thickness. An example (size-selected $\mathrm{WS}_{2}$ in aqueous SC) is shown in figure $2 \mathrm{~A} .{ }^{33}$ Systematic changes with nanosheet size are observed. The first thing to note is that shifts in the excitonic transitions are detected, likely as a result of electron confinement following exfoliation. Due to the scattering background, we recommend an analysis of the second derivative (figure 2B), where peak positions can be extracted more reliably. Please note that spectral smoothing is usually required to reduce the noise unless long integration times are used during the measurement. The appropriate smoothing is an important part of the data analysis and the smoothing method depends on the desired 
outcome. ${ }^{33}$ In this case, the second derivative was directly calculated from the spectra and afterwards smoothed with Adjacent Averaging.

As we have previously shown, edge effects result in a dependence of the spectral profile on nanosheet length in the absorbance and extinction spectra. ${ }^{34}$ The changes in spectral shape with nanosheet lateral size can be rationalized by edges being electronically distinct from centre regions. Therefore, the absorbance/extinction coefficient associated with the nanosheet edge is different from absorbance/extinction coefficients at the basal planes. This can be quantified via the ratio of extinction intensities at two different wavelengths. In principle, any peak intensity ratio can be related to the nanosheet size. However, the size metrics will be more reliable the larger the difference in the spectral shape at the given positions. An example is the peak intensity ratio at the high energy maximum over the local minimum as indicated in figure $2 \mathrm{~A}$ and plotted as a function of mean nanosheet size in figure $2 \mathrm{C} .{ }^{50}$ Interestingly, data for $\mathrm{MoS}_{2}$ and $\mathrm{WS}_{2}$ can collapse on the same curve if appropriate peak positions are chosen. This means that the peak intensity ratio for both materials can be quantitatively linked to the mean nanosheet length $\langle L\rangle$ via the same equation, which can be written as

$$
\langle L\rangle=\frac{2.30-E x t_{M a x-H E} / E x t_{M i n}}{0.02 E x t_{M a x-H E} / E x t_{M i n}-0.0185}
$$

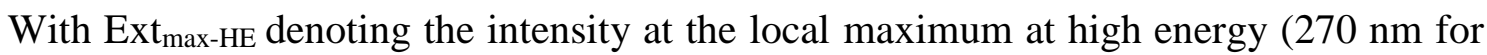
$\mathrm{MoS}_{2}$ and $235 \mathrm{~nm}$ for $\mathrm{WS}_{2}$ ) and Ext $\mathrm{Em}_{\text {min }}$ the extinction intensity at the local minimum (345 $\mathrm{nm}$ for $\mathrm{MoS}_{2}$ and $295 \mathrm{~nm}$ for $\mathrm{WS}_{2}$ ). In addition, confinement of the electrons in two dimensions results in changes in the band structure and hence shifts in the excitonic transitions. We note that additional effects such as the average dielectric constant may play an additional role making such correlations of the nanosheet thickness to exciton energies sensitive to the environment. Nonetheless, optical extinction spectra contain information on nanosheet thickness which can be quantified as illustrated in figure 2D, where the A-exciton energy is plotted as a function of mean nanosheet layer number $\langle N\rangle$ for $\mathrm{MoS}_{2}{ }^{34}$ and $\mathrm{WS}_{2},{ }^{33}$ which has been measured by AFM (see below). This empirical fitting of the data allows the nanosheet thickness (in aqueous SC dispersion media) to be extracted from equations 2 and 3.

$$
\begin{array}{ll}
N=2.3 \times 10^{36} e^{-54888 / \lambda_{A}}=2.3 \times 10^{36} e^{-44.3 E_{A}} & \text { (Eq. 2, } \left.\mathrm{MoS}_{2}\right) \\
N=6.35 \times 10^{-32} e^{\lambda_{A} / 8.51}=6.35 \times 10^{-32} e^{146 / E_{A}} & \text { (Eq. 3, } \left.\mathrm{WS}_{2}\right)
\end{array}
$$


In summary, this example shows that a simple measurement such as extinction spectroscopy contains information on nanosheet structure (and phase), concentration and potentially size and thickness. While we have reported that this is the case for a number of materials, a comprehensive understanding is certainly still lacking and requires future work.

Another widely utilised spectroscopic technique in this research area is Raman spectroscopy as it offers a unique fingerprint of the lattice vibrations and hence crystalline structure. ${ }^{61,88-96}$ The most common use of this technique for LPE materials is to verify whether the exfoliated material is structurally similar to the bulk material, i.e. that very few defects were introduced during processing. In this case, measurements are typically performed on filtered films or dried droplets of the LPE nanosheets. In these cases, spatial inhomogeneities in the deposited ensemble can lead to spot to spot variations in the obtained spectra, so that we recommend the recording of an average of at least 5-10 spectra in various regions. In severe cases it may be required to measure 100s of spectra.

In addition to an analysis of the Raman modes, standard Raman spectrometers can also be employed to measure photoluminescence (PL) spectra. For example, the PL emission of $\mathrm{WS}_{2}$ can be detected at 2000-3000 $\mathrm{cm}^{-1}$ Raman shift when excited using a green laser (532 $\mathrm{nm}){ }^{97}$ This situation is particularly useful for materials that are direct bandgap semiconductors when present as a monolayer and indirect semiconductors otherwise, as a Raman/PL measurement can then yield quantitative information on the monolayer content (if only the direct bandgap semiconductor is expected to show appreciable PL). ${ }^{33}$ In the following, we review how such measurements and quantification can be performed.

For the Raman/PL measurement of liquid dispersions to be successful, a number of details have to be encountered. Firstly, the measurement should be carried out using liquid dispersion rather than films, in order to avoid reaggregation and restacking in deposited material. This would clearly prevent the accurate quantification of monolayer content. From our experience, the most reliable results are obtained when measuring above the surface of a droplet of dispersion with a high concentration $\left(>0.3 \mathrm{gL}^{-1}\right)$. Note: such concentrations are easily accessible after LCC. The laser power during the measurement should be kept as low as possible to avoid sample heating and solvent evaporation. In fact, we were only able to carry out successful experiments in solvents with boiling points $>90^{\circ} \mathrm{C}$. Please also note that our reported measurements were all carried out in aqueous surfactant or polymer solution. ${ }^{33,73}$

As mentioned, it is crucial to focus the laser correctly when measuring Raman/PL on the high concentration dispersions. Usually, the camera image gives a good indication as 
shown in figure $2 \mathrm{E}$. The laser should be focused a minimum of $5 \mu \mathrm{m}$ above the surface of the droplet, as inner filter and reabsorption effects otherwise have a pronounced impact on the shape, position and intensity of the PL (at Raman shift 2000-3000 $\mathrm{cm}^{-1}$ when exciting WS 2 with $532 \mathrm{~nm}$ ) as seen in figure $2 \mathrm{~F}$. Examples of spectra of size selected $\mathrm{WS}_{2}$ with varying monolayer contents are displayed in figure $2 \mathrm{G}$ and show a systematic increase in the PL with increasing monolayer content, as expected. ${ }^{33}$ The ratio of the PL intensity to the main Raman mode intensity thus offers a metric to quantify the monolayer content in the dispersion as plotted in figure $2 \mathrm{H}$. While such a metric certainly offers exciting possibilities, we would like to note that it is currently only quantified for $\mathrm{WS}_{2}$ stabilized in aqueous media and excited with a $532 \mathrm{~nm}$ laser. Even in this case, it is unclear whether the quality of the starting material has an impact on the quantification or not. However, even without quantification, the monolayer content in the group VI TMDs is expected to always scale linearly with the $\mathrm{PL} /$ Raman ratio giving at least a semi-quantitative monolayer metric.
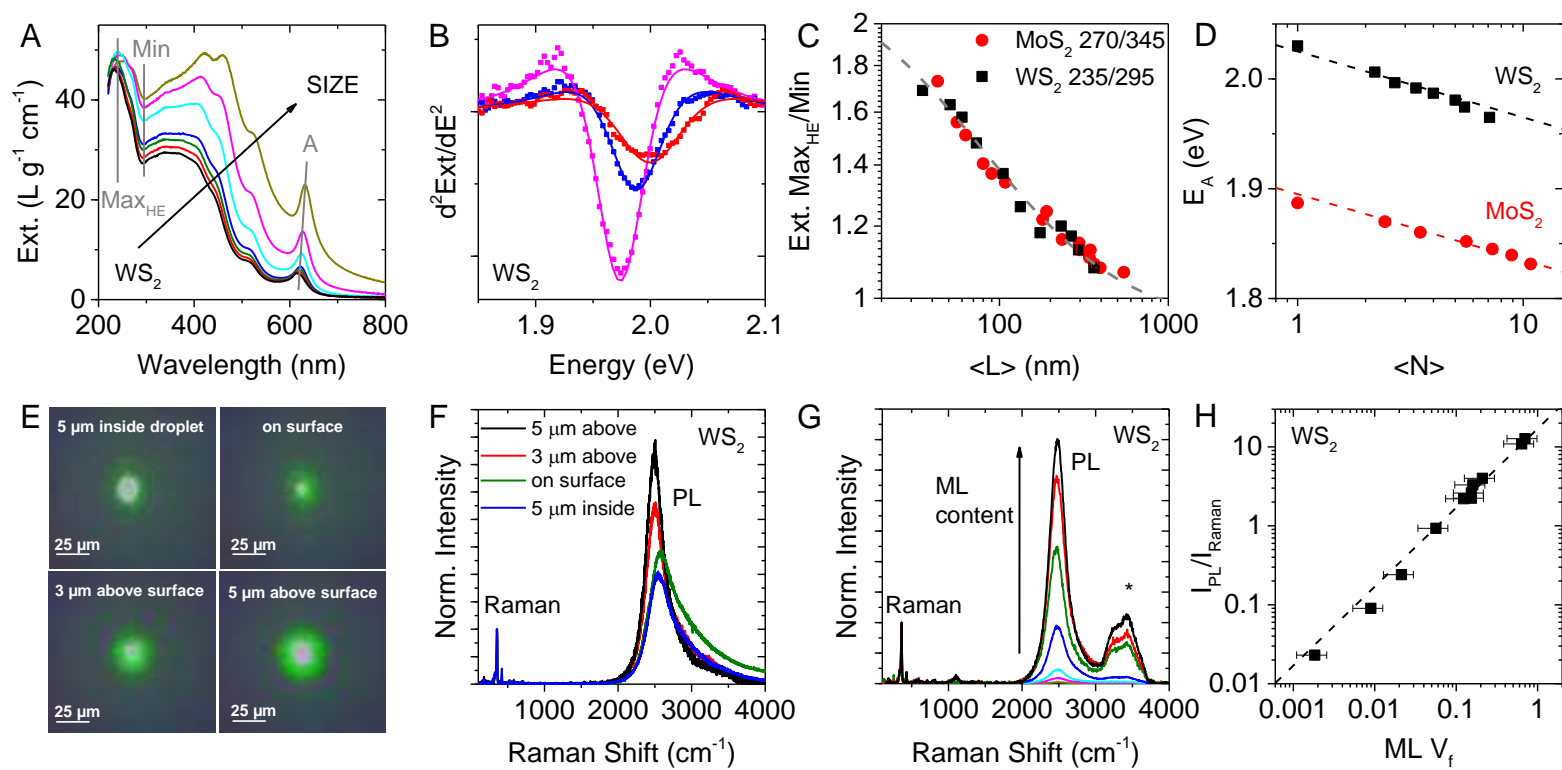

Figure 2: Basic spectroscopic characterization. A-D) UV-Vis spectroscopy. A) Optical extinction coefficient spectra of aqueous surfactant dispersions of size-selected $\mathrm{WS}_{2}$ showing systematic changes with nanosheet size. Peaks relevant for the analysis are indicated. B) Second derivatives of the A-exciton plotted versus energy for $\mathrm{WS}_{2}$ after smoothing the second derivative with Adjacent Averaging. The solid lines are fits to the second derivative of a Lorentzian to assess peak positions/energies. C) Plot of the peak intensity ratio at the high energy maximum / local minimum as a function of mean nanosheet length $\langle L\rangle$. Data for $\mathrm{MoS}_{2}$ and $\mathrm{WS}_{2}$ falls on the same curve. Hence the same equations can be used to quantify nanosheet length. D) Plot of A-exciton peak energies (from second derivatives) plotted as function of layer number $\langle N\rangle$ for $\mathrm{MoS}_{2}$ and $\mathrm{WS}_{2}$. E-H) Raman/PL measurements on high concentration dispersions. To obtain reliable results, it is important to focus the laser above the surface of the liquid drop to avoid inner-filter and reabsorption effects when the photoluminescence is to be analysed. The focusing of the laser is illustrated in E showing 
images of the laser focus inside the drop, on the surface and 3 and $5 \mu \mathrm{m}$ above the surface. The corresponding Raman/PL spectra (excitation $532 \mathrm{~nm}$ ) normalised to the $\mathrm{WS}_{2}$ Raman are displayed in F). The $\mathrm{WS}_{2}$ PL appears asymmetric, red-shifted, broadened and lower in intensity for incorrect laser focus. G) Normalised Raman/PL spectra (excitation $532 \mathrm{~nm}$ ) of size-selected $\mathrm{WS}_{2}$ showing systematic changes with nanosheet size and monolayer (ML) content. Spectra were recorded with the laser focused $>5 \mu \mathrm{m}$ above the surface. $\mathrm{H}$ ) $\mathrm{PL} /$ Raman intensity plotted as a function of monolayer volume fraction showing a linear relationship that can be used to determine the monolayer content.

\section{Microscopic characterization}

The spectroscopic characterization described above should always be complemented with microscopic characterization, even if spectroscopic size metrics are available to provide mean morphological characteristics of the produced nanosheets. In our laboratory, this is typically carried out using statistical transmission electron microscopy (TEM) and atomic force microscopy (AFM) for a subset of samples.

TEM is ideal for the analysis of nanosheet shape and lateral size; it provides higher resolution compared with scanning electron microscopy and higher throughput compared with AFM. To perform this measurement, the dispersion is drop-cast onto a TEM grid. Here, it is important to use dilute dispersions (i.e. optically transparent if the material absorbs in Vis region) to avoid reaggregation of the nanosheets during the deposition. Clearly, reaggregation is undesirable for the performance of precise length analysis. An example TEM image with ideal coverage and excessive nanosheet coverage is shown in figure $3 \mathrm{~A}$ and $\mathrm{B}$. Depending on the expected nanosheet size, continuous film grids can be beneficial to avoid small nanosheets falling through the holes. When drop-casting, the best results are obtained when the grid is placed on a filter paper to wick away access solvent. During the image acquisition, it is important to adjust the field of view according to the nanosheet size. If polydisperse dispersions are analysed, it is also required to record higher magnification images as to not bias the statistics towards larger, more easily discernible nanosheets in wide-view images. For the statistical length analysis, the longest dimension is measured and denoted as length and the direction perpendicular which is denoted as width. To obtain a robust mean value, at least 100 individually deposited nanosheets should be counted. Counting of fewer nanosheets may be sufficient for samples with smaller mean size, as these tend to be less polydisperse. If a non-size-selected stock dispersion is analyzed, it is recommended that at least 200 nanosheets should be recorded. 
Unfortunately, TEM is not well suited to the quantification of nanosheet layer number (and hence thickness) as this would require that all samples be suspended over vacuum (for example, to obtain electron energy loss spectra (EELS). In this situation, only nanosheets with large enough lateral dimensions would be available for measurement, hence providing a biased picture of the distribution of nanosheets that are present. This is illustrated by the representative TEM image of liquid-exfoliated $\mathrm{WS}_{2}$ shown in figure 3A. Accordingly, despite being more challenging and time consuming, we prefer to use AFM to determine the layer number of the exfoliated nanosheets.

For AFM analysis it is particularly critical to avoid re-aggregation of nanosheets on the wafer during solvent evaporation. For this, we drop-cast the dispersion on pre-heated $\mathrm{Si} / \mathrm{SiO}_{2}$ wafers $\left(10 \mu \mathrm{L}\right.$ per $0.5 \times 0.5 \mathrm{~cm}^{2}$ wafer). The solvent immediately evaporates and bubbles are formed, resulting in more uniform deposition compared to drop casting on wafers at lower temperatures. The wafer should be heated to $\sim 50-70^{\circ} \mathrm{C}$ above the boiling point of the solvent.

In the case of surfactant-based dispersions, it is recommended to dilute the sample with water (rather than surfactant) prior to deposition and wash the wafer thoroughly with water and IPA $(\sim 5 \mathrm{~mL}$ each) to remove residual surfactant. Residual surfactant can make the thickness measurements very tedious, especially for very small nanosheets that are more difficult to distinguish from surfactant. In this case, phase images can provide a guide as they usually give a good contrast between different materials. If problems with residual surfactant persist the wafers can be soaked in water overnight without significant loss of the nanosheets on the wafer. We generally find that deposition from high boiling point solvents such as NMP to be extremely challenging and we often observe pronounced reaggregation of the nanosheets and residual solvent or potentially polymerized NMP covering the nanosheets. We have recently overcome this problem by transferring the material exfoliated in NMP to IPA by high speed centrifugation prior to AFM. ${ }^{55}$

$\mathrm{Si} / \mathrm{SiO}_{2}$ wafers with a $200-300 \mathrm{~nm}$ oxide layer are recommended, as this enables nanoscaled objects to be seen with an optical microscope/optical zoom as blue spots. ${ }^{7}$ This is a useful guide to identify regions of interest for imaging, as is illustrated in figure 3B,C. Figure 3B shows optical micrographs in the case of a dispersion containing nanosheets with lateral size $>100 \mathrm{~nm}$. The area on the left is ideal for AFM analysis in terms of coverage, while the area on the right is too dense and mainly reaggregated nanosheets will be measured. In such cases further dilution is required prior to deposition on the wafer. When nanosheets are smaller they cannot be resolved as individual objects in optical micrographs. This is pictured 
in Figure 3C. Only reaggregated nanosheets will give a contrast in the optical image and such areas should be avoided. In these cases, we typically find region inside droplets to be promising as indicated by the dashed line in figure 3C. When using AFM it is also critical to adjust the field of view according to the nanosheet size. For example, the wide-view image in Figure 3D is not suitable for a precise statistical analysis and only higher magnification images such as the one in figure $3 \mathrm{E}$ will reveal the true size and thickness distribution of the nanosheets.

It is commonly found that measured, apparent AFM heights obtained using liquid exfoliated nanomaterials are overestimated due to the presence of residual solvent. In addition, accurate height measurements obtained using inhomogeneous samples (such as nanomaterials deposited on substrates) are generally challenging due to contributions from effects such as capillary forces and adhesion, which depend on the material and measurement parameters. ${ }^{98,99}$ To overcome these problems and obtain true thickness values from the apparent values measured by AFM, we and others have applied a procedure that we term step height analysis. 15, 27, 33, 34, 55, 63, 100 Here, incompletely exfoliated nanosheets showing clear terraces are first examined and the height of a number of steps are recorded. These terrace step heights will always be a multiple of the apparent thickness of one layer. Depending on the material, we and others have observed the apparent measured height of one layer of liquid-exfoliated nanosheets to be 1-2 $\mathrm{nm}$ which is much greater than the theoretical thickness. ${ }^{15,27,30,33,34,55,63}$ That the thinnest objects do indeed correspond to monolayers can be confirmed by Raman or PL analysis. ${ }^{15,34}$ The apparent monolayer height can then be used to convert the apparent measured AFM thickness to the layer number. For statistical analysis, again, we recommend that the height of at least 100-150 individually deposited nanosheets are recorded. If the thickness varies across the nanosheet, the mean value should be taken. From such statistical analysis, population histograms can be constructed. These histograms are typically log-normal in shape ${ }^{101}$ (also in the case of nanosheet length). If this is not the case the counting and/or imaging may be biased. From these histograms and the statistical analysis, the arithmetic number mean is obtained. This is typically also related to the volume fraction weighted mean value and therefore a valid measure of thickness. ${ }^{82}$ 

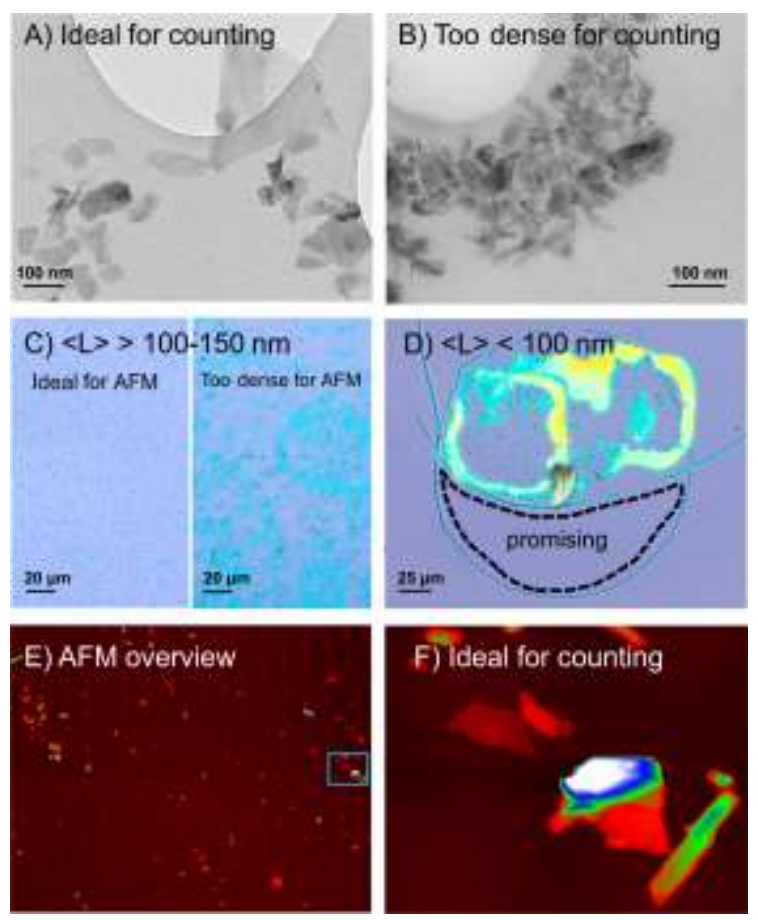

Figure 3: Basic microscopic characterization. A,B) Low resolution TEM images of liquidexfoliated $\mathrm{WS}_{2}$ with different nanosheet coverage. The image in A) has an ideal coverage for statistical size analysis, while the nanosheets in B) were drop-casted with a too high concentration and show pronounced reaggregation on the grid making statistical analysis impossible. C,D) Optical microscopic images of liquid-exfoliated $\mathrm{WS}_{2}$ drop-cast on $\mathrm{Si} / \mathrm{SiO}_{2}$ wafers (300 nm oxide). C) Nanosheets with sizes $>100-150 \mathrm{~nm}$ can be seen as little spots even at low magnification (such as typically available in AFMs), while smaller ones (D) only give contrast when they are aggregated. Regions of aggregates should be avoided for the AFM analysis. Promising regions are indicated in figure $C$ and D). E) Wide-view AFM image of a region such as the one shown in $\mathrm{C}$, left. Nanosheets of varying sizes and thicknesses are apparent. However, reliable size and thickness determination is not possible from such an image and requires zoom-in images such as shown in F).

\section{Film formation and printing}

To develop applications that make use of LPE nanosheets, it is necessary to convert the dispersed material into macroscopic structures that are suitable for further studies. In general, the nanosheets may either be deposited on a supporting substrate (such as glass, metal foil, silicon, or plastic film) or act as a filler within some host material (such as polymers, metals or other nanomaterials) to produce free-standing composites. To this end, numerous liquidphase processing techniques have been established that are very well suited to the deposition of nanosheet dispersions in combination with other dispersed nanomaterials or polymers. In this section we have outlined three approaches that are used in our laboratory to produce a range of nanosheet-containing structures and devices; vacuum filtration, ink-jet printing and spray deposition. 
Vacuum filtration is a convenient technique that is often used in our laboratory for the initial characterization of newly exfoliated nanosheet materials as well as for more detailed studies. This method involves the application of negative pressure using a pump to draw the liquid component of a dispersion through a porous polymer membrane and retain the dispersed solids. As a result, the dispersion contents are converted into a porous mat-like film on the membrane surface which is then available for transfer onto other substrates for testing. Although this technique is probably unsuitable for the production of nanomaterial-containing structures for most commercial purposes, its low cost and its simplicity makes it ideal for many research purposes. In particular, it enables excellent control over the composition of the resulting film via the constituents of the parent dispersion, as well as the deposited mass per unit area (and hence film thickness) via the volume of solvent that is passed through the membrane. By systematically varying these aspects of the produced films, we have studied the properties of a range of nanosheet containing systems such as graphene for transparent conductors, ${ }^{102}$ layered oxides combined with single walled carbon nanotubes (SWNTs) for supercapacitor electrodes, ${ }^{26,103}$ and TMDs/SWNT composites for electrocatalysis. ${ }^{104,} 105$

A wide range of filtration membranes available with different surface chemistries and pore-sizes, the choice of which will depend on the nature of the dispersion media, the size of the dispersed nanomaterials to be filtered and the desired approach to carry out film transfer. As the nanosheets that are produced by LPE often have lateral dimensions in the range of 10s to 100 s of nanometres, it is often necessary to use filtration membranes with smallest pore diameters possible. We frequently use mixed cellulose membranes with $25 \mathrm{~nm}$ pore size, as these are compatible with aqueous/surfactant dispersion media and the filtration membrane may be subsequently dissolved using acetone to achieve transfer of the filtered film. If the presence of excess surfactant is not desired in the final films, it can be useful to redisperse the nanosheet material at a lower surfactant concentration than was used during exfoliation steps to reduce its presence in subsequent film formation, or perform washing steps subsequent to film formation by simply passing additional water through the film.

To perform the transfer, the film-loaded membrane can be cut using a blade into the desired dimensions or conveniently into discs using a standard paper hole punch. The film is then positioned face down on the intended substrate and is then wetted using IPA. Pressure is applied by hand to ensure that good contact is made between the film and the underlying substrate and that no air bubbles are trapped in between them. Before the IPA has evaporated the combination is exposed to acetone vapour. Here a very small volume of acetone is heated 
using a hot plate in a conical flask to produce a vapour. The sample is held in the vapour for around 10 seconds, during which time the filtration membrane begins to dissolve due to condensation of the acetone vapour on the sample. The substrate is immediately immersed in an acetone bath that is refreshed every 10 to 15 minutes until the nitrocellulose is completely removed.

Despite the many advantages of vacuum filtration, the maximum film area is limited to the area of the filtration membrane and it also affords relatively limited control over the geometric features that can be transferred. As such, it is often preferable to use alternative deposition techniques, such spray deposition ${ }^{106}$ and ink-jet printing. ${ }^{36}$ The former allows for the production of highly uniform films with arbitrarily large lateral dimensions, while the latter enables greatly improved spatial control over the deposited material and is hence better suited to fabricate more complex structures.

The easy deposition of inks using low boiling point solvents such as water or IPA as carrier solvents can be achieved using an airbrush. Here, the ink is atomised at the nozzle of the airbrush by a nitrogen backpressure meaning rheological properties such as viscosity and surface energy play a small role in droplet formation. Using a heated platen allows a broader range of substrates (such as glass and silicon) to be used, as flash evaporation means the droplets do not have time to flow on the surface before evaporation. High concentration inks $\left(\sim 4 \mathrm{gL}^{-1}\right)$ are also easily deposited, as variable nozzle diameters $(125-600 \mu \mathrm{m})$ are available meaning large particles can be printed. The predominant limitation of the spray coating technique is its lack of precision; the airbrush deposits by continuous raster meaning substrates must be masked if a specific pattern is desired and a considerable amount of material is wasted. The need for masking means creating a horizontal heterostructure using this technique is extremely challenging.

To print a high resolution, heterostructured device, inkjet printing is the most precise method available. The Dimatix DMP 2300 printer has sixteen $21 \mu \mathrm{m}$ nozzles with droplets formed by a piezoelectric actuator. This means inks must be rheologically tuned to lie within a range specified by the inverse Ohnesorge number for satellite-free droplet formation. ${ }^{36} \mathrm{We}$ have used NMP as a carrier solvent in our recent work due to its excellent exfoliating and printing characteristics. ${ }^{45}$ This is convenient as materials such as graphene and $\mathrm{MoS}_{2}$ are stable in NMP and no additives are necessary to tune the rheology in order to jet the ink. NMP, however, has a high boiling point $\left(\sim 204^{\circ} \mathrm{C}\right)$ making it a challenge to be removed from printed films. Concentration limits place a further restraint on this method as an overly 
concentrated ink will result in blockage of the nozzle. We have experimentally found this limit to be $\sim 1.6 \mathrm{~g} \mathrm{~L}^{-1}$ for graphene flakes of $\sim 200 \mathrm{~nm}$ lateral flake dimensions.

\section{Concluding remarks and outlook}

In this work we have aimed to explain in detail the methodologies we use when producing and utilising liquid exfoliated nanosheets. We have discussed aspects of the production of nanosheet dispersions via exfoliation and described methods of characterization. Methods to process nanosheet dispersions into useful structures such as films and porous networks have been discussed. We have attempted to reach a finer degree of detail than is generally achieved in a research paper. It is our hope that this methods review will help new researchers to enter the area of liquid exfoliation and that as a result, the potential of these materials is more fully reached.

Despite the recent progress in liquid exfoliation, size selection, characterization and further processing, a number of challenges still need to be faced. For example, to further improve the exfoliation process, it will be critical to understand the role of the various process parameters on yield, nanosheet lateral size and thickness and monolayer content. With powerful high throughput spectroscopic metrics as the ones summarized above, such process optimization will be greatly facilitated in the future. Similarly, it will be important to devise and design new and efficient high yield size selection techniques, especially with regard to producing monolayer-rich samples with controlled lateral dimensions.

Furthermore, the more sophisticated liquid processing routes such as various printing techniques are still in their infancy for liquid-exfoliated nanosheets. For these strategies to be further improved, it will be critical to be able to tune the rheological properties of the dispersions, or inks on demand depending on the deposition technique to be used. An important part of this will be the investigation of new solvents and solvent blends.

Another remaining challenge will be efficient quality assessment and quality control of the exfoliated nanosheets. One can consider two main quality criteria: size (lateral size, thickness, monolayer content) and defectiveness of the nanosheets. While the former can be measured both microscopically and spectroscopically, a measure for the defect content remains elusive. In the case of graphene, Raman spectroscopy is probably the most common and most promising technique, as it can also distinguish between different nature of defects (such as defects at edges or on basal planes). ${ }^{107-109}$ Even though disorder is also reported to 
affect the Raman scattering for TMDs, ${ }^{90}$ a comprehensive understanding is still lacking in this case especially with regard to distinguishing between different kinds of defects with a high level of accuracy. For the group VI TMDs which are direct bandgap semiconductors in the monolayer, the excitonic line-width of the photoluminescence or photoluminescence quantum yield probably offers the potential to provide a metric for the defect content in the future. However, more work on fundamentally understanding the optical properties when systematically varying the defect content (or nanosheet lateral size), will be required.

Last but not least, it should be noted that in any case, the quality of the liquid-exfoliated nanosheets will always depend on the quality of the bulk starting materials. It will be of importance to compare different sources of bulk materials- both in terms of the exfoliation and basic characterisation itself as well as the resulting performance in relevant application areas. This emphasizes the demand for high quality starting materials which can only be met when equal research efforts are devoted to the synthesis of the bulk materials themselves.

Supporting Information. Tables of routinely used consumables and setups. This material is available free of charge via the Internet at http://pubs.acs.org.

\section{Author Contributions}

The manuscript was written through contributions of all authors. / All authors have given approval to the final version of the manuscript. / \$These authors contributed equally.

\section{Acknowledgement}

The research leading to these results has received funding from the European Union Seventh Framework Program under grant agreement n604391 Graphene Flagship. We have also received support from the Science Foundation Ireland (SFI) funded centre AMBER (SFI/12/RC/2278). In addition, JNC acknowledges the European Research Council (SEMANTICS) and SFI (11/PI/1087) for financial support and TMH acknowledges the European Union's Horizon 2020 research and innovation programme under the Marie Sklodowska-Curie grant agreement No. 707644. 


\section{References}

1. Chhowalla, M.; Shin, H. S.; Eda, G.; Li, L.-J.; Loh, K. P.; Zhang, H., The chemistry of twodimensional layered transition metal dichalcogenide nanosheets. Nature Chem. 2013, 5, 263-275.

2. Nicolosi, V.; Chhowalla, M.; Kanatzidis, M. G.; Strano, M. S.; Coleman, J. N., Liquid Exfoliation of Layered Materials. Science 2013, 340, 1420.

3. Wang, Q. H.; Kalantar-Zadeh, K.; Kis, A.; Coleman, J. N.; Strano, M. S., Electronics and optoelectronics of two-dimensional transition metal dichalcogenides. Nat. Nanotechnol. 2012, 7, 699712.

4. Osada, M.; Sasaki, T., Exfoliated oxide nanosheets: new solution to nanoelectronics. J. Mater. Chem. 2009, 19, 2503-2511.

5. Ray, S. S.; Okamoto, M., Polymer/layered silicate nanocomposites: a review from preparation to processing. Prog. Polym. Sci. 2003, 28, 1539-1641.

6. Novoselov, K. S.; Fal'ko, V. I.; Colombo, L.; Gellert, P. R.; Schwab, M. G.; Kim, K., A roadmap for graphene. Nature 2012, 490, 192-200.

7. Novoselov, K. S.; Jiang, D.; Schedin, F.; Booth, T. J.; Khotkevich, V. V.; Morozov, S. V.; Geim, A. K., Two-dimensional atomic crystals. Proc. Natl. Acad. Sci. U. S. A. 2005, 102, 1045110453.

8. Reina, A.; Jia, X. T.; Ho, J.; Nezich, D.; Son, H. B.; Bulovic, V.; Dresselhaus, M. S.; Kong, J., Large Area, Few-Layer Graphene Films on Arbitrary Substrates by Chemical Vapor Deposition. Nano Lett. 2009, 9, 30-35.

9. Gatensby, R.; McEvoy, N.; Lee, K.; Hallam, T.; Berner, N. C.; Rezvani, E.; Winters, S.; O'Brien, M.; Duesberg, G. S., Controlled synthesis of transition metal dichalcogenide thin films for electronic applications. Appl. Surf. Sci. 2014, 297, 139-146.

10. Dreyer, D. R.; Park, S.; Bielawski, C. W.; Ruoff, R. S., The chemistry of graphene oxide. Chem. Soc. Rev. 2010, 39, 228-240.

11. Parvez, K.; Wu, Z. S.; Li, R. J.; Liu, X. J.; Graf, R.; Feng, X. L.; Mullen, K., Exfoliation of Graphite into Graphene in Aqueous Solutions of Inorganic Salts. J. Am. Chem. Soc. 2014, 136, 60836091. 
12. Abellán, G.; Martí-Gastaldo, C.; Ribera, A.; Coronado, E., Hybrid Materials Based on Magnetic Layered Double Hydroxides: A Molecular Perspective. Acc. Chem. Res. 2015, 48, 16011611.

13. Coleman, J. N.; Lotya, M.; O'Neill, A.; Bergin, S. D.; King, P. J.; Khan, U.; Young, K.; Gaucher, A.; De, S.; Smith, R. J.; Shvets, I. V.; Arora, S. K.; Stanton, G.; Kim, H.-Y.; Lee, K.; Kim, G. T.; Duesberg, G. S.; Hallam, T.; Boland, J. J.; Wang, J. J.; Donegan, J. F.; Grunlan, J. C.; Moriarty, G.; Shmeliov, A.; Nicholls, R. J.; Perkins, J. M.; Grieveson, E. M.; Theuwissen, K.; McComb, D. W.; Nellist, P. D.; Nicolosi, V., Two-Dimensional Nanosheets Produced by Liquid Exfoliation of Layered Materials. Science 2011, 331, 568-571.

14. Hernandez, Y.; Nicolosi, V.; Lotya, M.; Blighe, F. M.; Sun, Z.; De, S.; McGovern, I. T.; Holland, B.; Byrne, M.; Gun'Ko, Y. K.; Boland, J. J.; Niraj, P.; Duesberg, G.; Krishnamurthy, S.; Goodhue, R.; Hutchison, J.; Scardaci, V.; Ferrari, A. C.; Coleman, J. N., High-yield production of graphene by liquid-phase exfoliation of graphite. Nat. Nanotechnol. 2008, 3, 563-568.

15. Paton, K. R.; Varrla, E.; Backes, C.; Smith, R. J.; Khan, U.; O'Neill, A.; Boland, C.; Lotya, M.; Istrate, O. M.; King, P.; Higgins, T.; Barwich, S.; May, P.; Puczkarski, P.; Ahmed, I.; Moebius, M.; Pettersson, H.; Long, E.; Coelho, J.; O'Brien, S. E.; McGuire, E. K.; Sanchez, B. M.; Duesberg, G. S.; McEvoy, N.; Pennycook, T. J.; Downing, C.; Crossley, A.; Nicolosi, V.; Coleman, J. N., Scalable production of large quantities of defect-free few-layer graphene by shear exfoliation in liquids. Nat. Mater. 2014, 13, 624-630.

16. Cunningham, G.; Lotya, M.; Cucinotta, C. S.; Sanvito, S.; Bergin, S. D.; Menzel, R.; Shaffer, M. S. P.; Coleman, J. N., Solvent Exfoliation of Transition Metal Dichalcogenides: Dispersibility of Exfoliated Nanosheets Varies Only Weakly between Compounds. ACS Nano 2012, 6, 3468-3480.

17. Smith, R. J.; King, P. J.; Lotya, M.; Wirtz, C.; Khan, U.; De, S.; O'Neill, A.; Duesberg, G. S.; Grunlan, J. C.; Moriarty, G.; Chen, J.; Wang, J. Z.; Minett, A. I.; Nicolosi, V.; Coleman, J. N., LargeScale Exfoliation of Inorganic Layered Compounds in Aqueous Surfactant Solutions. Adv. Mater. 2011, 23, 3944-3948. 
18. May, P.; Khan, U.; Hughes, J. M.; Coleman, J. N., Role of Solubility Parameters in Understanding the Steric Stabilization of Exfoliated Two-Dimensional Nanosheets by Adsorbed Polymers. J. Phys. Chem. C 2012, 116, 11393-11400.

19. Coleman, J. N., Liquid Exfoliation of Defect-Free Graphene. Acc. Chem. Res. 2013, 46, 14-22.

20. Khan, U.; Porwal, H.; O'Neill, A.; Nawaz, K.; May, P.; Coleman, J. N., Solvent-exfoliated graphene at extremely high concentration. Langmuir 2011, 27, 9077-82.

21. Liu, L. H.; Zorn, G.; Castner, D. G.; Solanki, R.; Lerner, M. M.; Yan, M. D., A simple and scalable route to wafer-size patterned graphene. J. Mater. Chem. 2010, 20, 5041-5046.

22. Du, W. C.; Lu, J.; Sun, P. P.; Zhu, Y. Y.; Jiang, X. Q., Organic salt-assisted liquid-phase exfoliation of graphite to produce high-quality graphene. Chem. Phys. Lett. 2013, 568, 198-201.

23. Zhi, C. Y.; Bando, Y.; Tang, C. C.; Kuwahara, H.; Golberg, D., Large-Scale Fabrication of Boron Nitride Nanosheets and Their Utilization in Polymeric Composites with Improved Thermal and Mechanical Properties. Adv. Mater. 2009, 21, 2889-2893.

24. Bang, G. S.; Nam, K. W.; Kim, J. Y.; Shin, J.; Choi, J. W.; Choi, S. Y., Effective Liquid-Phase Exfoliation and Sodium Ion Battery Application of MoS2 Nanosheets. ACS Appl. Mater. Interfaces 2014, 6, 7084-7089.

25. Alsaif, M.; Balendhran, S.; Field, M. R.; Latham, K.; Wlodarski, W.; Ou, J. Z.; KalantarZadeh, K., Two dimensional alpha-MoO3 nanoflakes obtained using solvent-assisted grinding and sonication method: Application for H-2 gas sensing. Sens. Actuators, B 2014, 192, 196-204.

26. Hanlon, D.; Backes, C.; Higgins, T. M.; Hughes, M.; O’Neill, A.; King, P.; McEvoy, N.; Duesberg, G. S.; Mendoza Sanchez, B.; Pettersson, H.; Nicolosi, V.; Coleman, J. N., Production of Molybdenum Trioxide Nanosheets by Liquid Exfoliation and Their Application in High-Performance Supercapacitors. Chem. Mater. 2014, 26, 1751-1763.

27. Harvey, A.; Backes, C.; Gholamvand, Z.; Hanlon, D.; McAteer, D.; Nerl, H. C.; McGuire, E.; Seral-Ascaso, A.; Ramasse, Q. M.; McEvoy, N.; Winters, S.; Berner, N. C.; McCloskey, D.; Donegan, J. F.; Duesberg, G. S.; Nicolosi, V.; Coleman, J. N., Preparation of Gallium Sulfide Nanosheets by Liquid Exfoliation and Their Application As Hydrogen Evolution Catalysts. Chem. Mater. 2015, 27, 3483-3493. 
28. Kang, J.; Wood, J. D.; Wells, S. A.; Lee, J.-H.; Liu, X.; Chen, K.-S.; Hersam, M. C., Solvent Exfoliation of Electronic-Grade, Two-Dimensional Black Phosphorus. ACS Nano 2015, 9, 3596-3604. 29. Yasaei, P.; Kumar, B.; Foroozan, T.; Wang, C.; Asadi, M.; Tuschel, D.; Indacochea, J. E.; Klie, R. F.; Salehi-Khojin, A., High-Quality Black Phosphorus Atomic Layers by Liquid-Phase Exfoliation. Adv. Mater. 2015, 27, 1887-1892.

30. Harvey, A.; He, X.; Godwin, I. J.; Backes, C.; McAteer, D.; Berner, N. C.; McEvoy, N.; Ferguson, A.; Shmeliov, A.; Lyons, M. E. G.; Nicolosi, V.; Duesberg, G. S.; Donegan, J. F.; Coleman, J. N., Production of $\mathrm{Ni}(\mathrm{OH}) 2$ nanosheets by liquid phase exfoliation: from optical properties to electrochemical applications. J. Mater. Chem. C. 2016, 4, 11046-11059.

31. Naguib, M.; Mashtalir, O.; Carle, J.; Presser, V.; Lu, J.; Hultman, L.; Gogotsi, Y.; Barsoum, M. W., Two-Dimensional Transition Metal Carbides. ACS Nano 2012, 6, 1322-1331.

32. Yi, M.; Shen, Z., Kitchen blender for producing high-quality few-layer graphene. Carbon 2014, 78, 622-626.

33. Backes, C.; Szydłowska, B. M.; Harvey, A.; Yuan, S.; Vega-Mayoral, V.; Davies, B. R.; Zhao, P.-1.; Hanlon, D.; Santos, E. J. G.; Katsnelson, M. I.; Blau, W. J.; Gadermaier, C.; Coleman, J. N., Production of Highly Monolayer Enriched Dispersions of Liquid-Exfoliated Nanosheets by Liquid Cascade Centrifugation. ACS Nano 2016, 10 1589-1601.

34. Backes, C.; Smith, R. J.; McEvoy, N.; Berner, N. C.; McCloskey, D.; Nerl, H. C.; O’Neill, A.; King, P. J.; Higgins, T.; Hanlon, D.; Scheuschner, N.; Maultzsch, J.; Houben, L.; Duesberg, G. S.; Donegan, J. F.; Nicolosi, V.; Coleman, J. N., Edge and Confinement Effects Allow in situ Measurement of Size and Thickness of Liquid-Exfoliated Nanosheets. Nature Commun. 2014, 5, 4576.

35. Mendoza-Sanchez, B.; Coelho, J.; Pokle, A.; Nicolosi, V., A 2D graphene-manganese oxide nanosheet hybrid synthesized by a single step liquid-phase co-exfoliation method for supercapacitor applications. Electrochim. Acta 2015, 174, 696-705.

36. Finn, D. J.; Lotya, M.; Cunningham, G.; Smith, R. J.; McCloskey, D.; Donegan, J. F.; Coleman, J. N., Inkjet deposition of liquid-exfoliated graphene and MoS2 nanosheets for printed device applications. J. Mater. Chem. C 2014, 2, 925-932. 
37. Torrisi, F.; Hasan, T.; Wu, W. P.; Sun, Z. P.; Lombardo, A.; Kulmala, T. S.; Hsieh, G. W.; Jung, S. J.; Bonaccorso, F.; Paul, P. J.; Chu, D. P.; Ferrari, A. C., Inkjet-Printed Graphene Electronics. ACS Nano 2012, 6, 2992-3006.

38. Zhang, X. T.; Sui, Z. Y.; Xu, B.; Yue, S. F.; Luo, Y. J.; Zhan, W. C.; Liu, B., Mechanically strong and highly conductive graphene aerogel and its use as electrodes for electrochemical power sources. J. Mater. Chem. 2011, 21, 6494-6497.

39. Sun, J.; Lee, H.-W.; Pasta, M.; Yuan, H.; Zheng, G.; Sun, Y.; Li, Y.; Cui, Y., A phosphorenegraphene hybrid material as a high-capacity anode for sodium-ion batteries. Nat. Nanotechnol. 2015, $10,980-985$

40. Biscarat, J.; Bechelany, M.; Pochat-Bohatier, C.; Miele, P., Graphene-like BN/gelatin nanobiocomposites for gas barrier applications. Nanoscale 2015, 7, 613-618.

41. Xie, S.; Istrate, O. M.; May, P.; Barwich, S.; Bell, A. P.; Khana, U.; Coleman, J. N., Boron nitride nanosheets as barrier enhancing fillers in melt processed composites. Nanoscale 2015, 7, 44434450.

42. Cunningham, G.; Hanlon, D.; McEvoy, N.; Duesberg, G. S.; Coleman, J. N., Large variations in both dark- and photoconductivity in nanosheet networks as nanomaterial is varied from MoS2 to WTe2. Nanoscale 2014, 7, 198-208.

43. Withers, F.; Yang, H.; Britnell, L.; Rooney, A. P.; Lewis, E.; Felten, A.; Woods, C. R.; Sanchez Romaguera, V.; Georgiou, T.; Eckmann, A.; Kim, Y. J.; Yeates, S. G.; Haigh, S. J.; Geim, A. K.; Novoselov, K. S.; Casiraghi, C., Heterostructures Produced from Nanosheet-Based Inks. Nano Lett. 2014, 14, 3987-3992.

44. Varrla, E.; Paton, K. R.; Backes, C.; Harvey, A.; Smith, R. J.; McCauley, J.; Coleman, J. N., Turbulence-assisted shear exfoliation of graphene using household detergent and a kitchen blender. Nanoscale 2014, 6, 11810-11819.

45. Bonaccorso, F.; Bartolotta, A.; Coleman, J. N.; Backes, C., 2D-Crystal-Based Functional Inks. Adv. Mater. 2016, 28, 6136-6166.

46. Ciesielski, A.; Samori, P., Graphene via sonication assisted liquid-phase exfoliation. Chem. Soc. Rev. 2014, 43, 381-398. 
47. Parvez, K.; Yang, S.; Feng, X.; Müllen, K., Exfoliation of graphene via wet chemical routes. Synth. Met. 2015, 210, Part A, 123-132.

48. Yi, M.; Shen, Z., A review on mechanical exfoliation for the scalable production of graphene. J. Mater. Chem. C. 2015, 3, 11700-11715.

49. Yi, M.; Shen, Z., Fluid dynamics: an emerging route for the scalable production of graphene in the last five years. $R S C A d v$. 2016, 6, 72525-72536.

50. Backes, C.; Hanlon, D.; Szydlowska, B. M.; Harvey, A.; Smith, R. J.; Higgins, T. M.; Coleman, J. N., Preparation of liquid-exfoliated transition metal dichalcogenide nanosheets with controlled size and thickness. J. Vis. Exp. 2016, accepted, doi:10.3791/54806.

51. Hughes, J. M.; Aherne, D.; Coleman, J. N., Generalizing solubility parameter theory to apply to one- and two-dimensional solutes and to incorporate dipolar interactions. J. Appl. Polym. Sci. 2013, $127,4483-4491$.

52. Shen, J.; He, Y.; Wu, J.; Gao, C.; Keyshar, K.; Zhang, X.; Yang, Y.; Ye, M.; Vajtai, R.; Lou, J.; Ajayan, P. M., Liquid Phase Exfoliation of Two-Dimensional Materials by Directly Probing and Matching Surface Tension Components. Nano Lett. 2015, 15, 5449-5454.

53. Kim, J.; Kwon, S.; Cho, D.-H.; Kang, B.; Kwon, H.; Kim, Y.; Park, S. O.; Jung, G. Y.; Shin, E.; Kim, W.-G.; Lee, H.; Ryu, G. H.; Choi, M.; Kim, T. H.; Oh, J.; Park, S.; Kwak, S. K.; Yoon, S. W.; Byun, D.; Lee, Z.; Lee, C., Direct exfoliation and dispersion of two-dimensional materials in pure water via temperature control. Nature Commun. 2015, 6, 9294.

54. Smith, R. J.; Lotya, M.; Coleman, J. N., The importance of repulsive potential barriers for the dispersion of graphene using surfactants. New J. Phys. 2010, 12, 125008.

55. Hanlon, D.; Backes, C.; Doherty, E.; Cucinotta, C. S.; Berner, N. C.; Boland, C.; Lee, K.; Lynch, P.; Gholamvand, Z.; Harvey, A.; Zhang, S.; Wang, K.; Moynihan, G.; Pokle, A.; Ramasse, Q. M.; McEvoy, N.; Blau, W. J.; Wang, J.; Abellan, G.; Hauke, F.; Hirsch, A.; Sanvito, S.; O’Regan, D. D.; Duesberg, G. S.; Nicolosi, V.; Coleman, J. N., Liquid Exfoliation of Solvent-Stabilised Few-Layer Black Phosphorus for Applications Beyond Electronics. Nature Commun. 2015, 6, 8563. 
56. Varrla, E.; Backes, C.; Paton, K. R.; Harvey, A.; Gholamvand, Z.; McCauley, J.; Coleman, J. N., Large-Scale Production of Size-Controlled MoS2 Nanosheets by Shear Exfoliation. Chem. Mater. 2015, 27, 1129-1139.

57. Khan, U.; O'Neill, A.; Lotya, M.; De, S.; Coleman, J. N., High-Concentration Solvent Exfoliation of Graphene. Small 2010, 6, 864-871.

58. Lotya, M.; King, P. J.; Khan, U.; De, S.; Coleman, J. N., High-Concentration, SurfactantStabilized Graphene Dispersions. ACS Nano 2010, 4, 3155-3162.

59. Green, A. A.; Hersam, M. C., Solution Phase Production of Graphene with Controlled Thickness via Density Differentiation. Nano Lett. 2009, 9, 4031-4036.

60. Paredes, J. I.; Villar-Rodil, S., Biomolecule-assisted exfoliation and dispersion of graphene and other two-dimensional materials: a review of recent progress and applications. Nanoscale 2016, 8, 15389-15413.

61. Favron, A.; Gaufres, E.; Fossard, F.; Phaneuf-Lheureux, A.-L.; Tang, N. Y. W.; Levesque, P. L.; Loiseau, A.; Leonelli, R.; Francoeur, S.; Martel, R., Photooxidation and quantum confinement effects in exfoliated black phosphorus. Nat. Mater. 2015, 14, 826-832.

62. Haar, S.; Bruna, M.; Lian, J. X.; Tomarchio, F.; Olivier, Y.; Mazzaro, R.; Morandi, V.; Moran, J.; Ferrari, A. C.; Beljonne, D.; Ciesielski, A.; Samorì, P., Liquid-Phase Exfoliation of Graphite into Single- and Few-Layer Graphene with $\alpha$-Functionalized Alkanes. J. Phys. Chem. Lett. 2016, 7, 27142721.

63. Fan, X.; Xu, P.; Li, Y. C.; Zhou, D.; Sun, Y.; Nguyen, M. A. T.; Terrones, M.; Mallouk, T. E., Controlled Exfoliation of MoS2 Crystals into Trilayer Nanosheets. J. Am. Chem. Soc. 2016, 138, $5143-5149$.

64. Capasso, A.; Del Rio Castillo, A. E.; Sun, H.; Ansaldo, A.; Pellegrini, V.; Bonaccorso, F., Inkjet printing of graphene for flexible electronics: An environmentally-friendly approach. Solid State Commun. 2015, 224, 53-63.

65. Zhou, K.-G.; Mao, N.-N.; Wang, H.-X.; Peng, Y.; Zhang, H.-L., A Mixed-Solvent Strategy for Efficient Exfoliation of Inorganic Graphene Analogues. Angew. Chem. Int. Ed. 2011, 50, 1083910842. 
66. Azani, M.-R.; Hassanpour, A.; Carcelén, V.; Gibaja, C.; Granados, D.; Mas-Ballesté, R.; Zamora, F., Highly concentrated and stable few-layers graphene suspensions in pure and volatile organic solvents. Appl. Mater. Today 2016, 2, 17-23.

67. Min, Y.; Zhigang, S.; Xiaojing, Z.; Shulin, M., Achieving concentrated graphene dispersions in water/acetone mixtures by the strategy of tailoring Hansen solubility parameters. J. Phys. D: Appl. Phys. 2013, 46, 025301.

68. Gravagnuolo, A. M.; Morales-Narváez, E.; Longobardi, S.; da Silva, E. T.; Giardina, P.; Merkoçi, A., In Situ Production of Biofunctionalized Few-Layer Defect-Free Microsheets of Graphene. Adv. Funct. Mater. 2015, 25, 2771-2779.

69. Guan, G.; Zhang, S.; Liu, S.; Cai, Y.; Low, M.; Teng, C. P.; Phang, I. Y.; Cheng, Y.; Duei, K. L.; Srinivasan, B. M.; Zheng, Y.; Zhang, Y.-W.; Han, M.-Y., Protein Induces Layer-by-Layer Exfoliation of Transition Metal Dichalcogenides. J. Am. Chem. Soc. 2015, 137, 6152-6155.

70. Kang, J.; Seo, J.-W. T.; Alducin, D.; Ponce, A.; Yacaman, M. J.; Hersam, M. C., Thickness sorting of two-dimensional transition metal dichalcogenides via copolymer-assisted density gradient ultracentrifugation. Nature Commun. 2014, 5.

71. Zhu, J.; Kang, J.; Kang, J.; Jariwala, D.; Wood, J. D.; Seo, J.-W. T.; Chen, K.-S.; Marks, T. J.; Hersam, M. C., Solution-Processed Dielectrics Based on Thickness-Sorted Two-Dimensional Hexagonal Boron Nitride Nanosheets. Nano Lett. 2015, 15, 7029-7036.

72. May, P.; Khan, U.; Hughes, J. M.; Coleman, J. N., Role of Solubility Parameters in Understanding the Steric Stabilization of Exfoliated Two-Dimensional Nanosheets by Adsorbed Polymers. J. Phys. Chem. C 2012, 116, 11393-11400.

73. Vega-Mayoral, V.; Backes, C.; Hanlon, D.; Khan, U.; Gholamvand, Z.; O'Brien, M.; Duesberg, G. S.; Gadermaier, C.; Coleman, J. N., Photoluminescence from Liquid-Exfoliated WS2 Monomers in Poly(Vinyl Alcohol) Polymer Composites. Adv. Funct. Mater. 2016, 26, 1028-1039.

74. Hernandez, Y.; Lotya, M.; Rickard, D.; Bergin, S. D.; Coleman, J. N., Measurement of Multicomponent Solubility Parameters for Graphene Facilitates Solvent Discovery. Langmuir 2010, 26, 3208-3213. 
75. Damm, C.; Nacken, T. J.; Peukert, W., Quantitative evaluation of delamination of graphite by wet media milling. Carbon 2015, 81, 284-294.

76. Knieke, C.; Berger, A.; Voigt, M.; Taylor, R. N. K.; Röhrl, J.; Peukert, W., Scalable production of graphene sheets by mechanical delamination. Carbon 2010, 48, 3196-3204.

77. Leon, V.; Quintana, M.; Herrero, M. A.; Fierro, J. L. G.; Hoz, A. d. 1.; Prato, M.; Vazquez, E., Few-layer graphenes from ball-milling of graphite with melamine. Chem. Commun. 2011, 47, 1093610938.

78. Nacken, T. J.; Damm, C.; Xing, H.; Rüger, A.; Peukert, W., Determination of quantitative structure-property and structure-process relationships for graphene production in water. Nano Res. 2015, 8, 1865-1881.

79. Yi, M.; Shen, Z.; Zhang, W.; Zhu, J.; Liu, L.; Liang, S.; Zhang, X.; Ma, S., Hydrodynamicsassisted scalable production of boron nitride nanosheets and their application in improving oxygenatom erosion resistance of polymeric composites. Nanoscale 2013, 5, 10660-10667.

80. Liu, L.; Shen, Z.; Yi, M.; Zhang, X.; Ma, S., A green, rapid and size-controlled production of high-quality graphene sheets by hydrodynamic forces. RSC Adv. 2014, 4, 36464-36470.

81. Liang, S.; Yi, M.; Shen, Z.; Liu, L.; Zhang, X.; Ma, S., One-step green synthesis of graphene nanomesh by fluid-based method. RSC Adv. 2014, 4, 16127-16131.

82. Backes, C.; Paton, K. R.; Hanlon, D.; Yuan, S.; Katsnelson, M. I.; Houston, J.; Smith, R. J.; McCloskey, D.; Donegan, J. F.; Coleman, J. N., Spectroscopic metrics allow in situ measurement of mean size and thickness of liquid-exfoliated few-layer graphene nanosheets. Nanoscale 2016, 8, 43114323.

83. Wilson, J. A.; Yoffe, A. D., Transition metal dichalcogenides. Discussion and interpretation of the observed optical, electrical, and structural properties. Adv. Phys. 1969, 18, 193-335.

84. Eda, G.; Yamaguchi, H.; Voiry, D.; Fujita, T.; Chen, M.; Chhowalla, M., Photoluminescence from Chemically Exfoliated MoS2. Nano Lett. 2011, 11, 5111-5116.

85. Knirsch, K. C.; Berner, N. C.; Nerl, H. C.; Cucinotta, C. S.; Gholamvand, Z.; McEvoy, N.; Wang, Z.; Abramovic, I.; Vecera, P.; Halik, M.; Sanvito, S.; Duesberg, G. S.; Nicolosi, V.; Hauke, F.; 
Hirsch, A.; Coleman, J. N.; Backes, C., Basal-plane functionalisation of chemically-exfoliated molybdenum disulfide by diazonium salts. ACS Nano 2015, 9, 6018-6030.

86. Paton, K. R.; Coleman, J. N., Relating the optical absorption coefficient of nanosheet dispersions to the intrinsic monolayer absorption. Carbon 2016, 107, 733-738.

87. Yadgarov, L.; Choi, C. L.; Sedova, A.; Cohen, A.; Rosentsveig, R.; Bar-Elli, O.; Oron, D.;

Dai, H.; Tenne, R., Dependence of the Absorption and Optical Surface Plasmon Scattering of MoS2 Nanoparticles on Aspect Ratio, Size, and Media. ACS Nano 2014, 8, 3575-3583.

88. Li, H.; Zhang, Q.; Yap, C. C. R.; Tay, B. K.; Edwin, T. H. T.; Olivier, A.; Baillargeat, D., From Bulk to Monolayer MoS2: Evolution of Raman Scattering. Adv. Funct. Mater. 2012, 22, 13851390.

89. Thripuranthaka, M.; Kashid, R. V.; Sekhar Rout, C.; Late, D. J., Temperature dependent Raman spectroscopy of chemically derived few layer MoS2 and WS2 nanosheets. Appl. Phys. Lett. 2014, 104, 081911

90. Mignuzzi, S.; Pollard, A. J.; Bonini, N.; Brennan, B.; Gilmore, I. S.; Pimenta, M. A.; Richards, D.; Roy, D., Effect of disorder on Raman scattering of single-layer MoS2. Phys. Rev. B 2015, 91, 195411.

91. Pimenta, M. A.; del Corro, E.; Carvalho, B. R.; Fantini, C.; Malard, L. M., Comparative Study of Raman Spectroscopy in Graphene and MoS2-type Transition Metal Dichalcogenides. Acc. Chem. Res. 2015, 48, 41-47.

92. Berkdemir, A.; Gutierrez, H. R.; Botello-Mendez, A. R.; Perea-Lopez, N.; Elias, A. L.; Chia, C.-I.; Wang, B.; Crespi, V. H.; Lopez-Urias, F.; Charlier, J.-C.; Terrones, H.; Terrones, M., Identification of individual and few layers of WS2 using Raman Spectroscopy. Sci. Rep. 2013, 3.

93. Saito, R.; Tatsumi, Y.; Huang, S.; Ling, X.; Dresselhaus, M. S., Raman spectroscopy of transition metal dichalcogenides. J. Phys.: Condens. Matter 2016, 28, 353002.

94. Maria, O. B.; Niall, M.; Carlo, M.; Jian-Yao, Z.; Nina, C. B.; Jani, K.; Kenan, E.; Timothy, J. P.; Jannik, C. M.; Chanyoung, Y.; Mohamed, A.; Toby, H.; John, F. D.; Stefano, S.; Georg, S. D., Raman characterization of platinum diselenide thin films. 2D Materials 2016, 3, 021004. 
95. Ferrari, A. C.; Basko, D. M., Raman spectroscopy as a versatile tool for studying the properties of graphene. Nat. Nanotechnol. 2013, 8, 235-246.

96. Zhang, X.; Qiao, X.-F.; Shi, W.; Wu, J.-B.; Jiang, D.-S.; Tan, P.-H., Phonon and Raman scattering of two-dimensional transition metal dichalcogenides from monolayer, multilayer to bulk material. Chem. Soc. Rev. 2015, 44, 2757-2785.

97. Gutiérrez, H. R.; Perea-López, N.; Elías, A. L.; Berkdemir, A.; Wang, B.; Lv, R.; LópezUrías, F.; Crespi, V. H.; Terrones, H.; Terrones, M., Extraordinary Room-Temperature Photoluminescence in Triangular WS2 Monolayers. Nano Lett. 2012, 13, 3447-3454.

98. Ridings, C.; Warr, G. G.; Andersson, G. G., Composition of the outermost layer and concentration depth profiles of ammonium nitrate ionic liquid surfaces. Phys. Chem. Chem. Phys. 2012, 14, 16088-16095.

99. Nemes-Incze, P.; Osváth, Z.; Kamarás, K.; Biró, L. P., Anomalies in thickness measurements of graphene and few layer graphite crystals by tapping mode atomic force microscopy. Carbon 2008, $46,1435-1442$.

100. Gibaja, C.; Rodriguez-San-Miguel, D.; Ares, P.; Gómez-Herrero, J.; Varela, M.; Gillen, R.; Maultzsch, J.; Hauke, F.; Hirsch, A.; Abellán, G.; Zamora, F., Few-Layer Antimonene by LiquidPhase Exfoliation. Angew. Chem. Int. Ed. 2016, n/a-n/a.

101. Kouroupis-Agalou, K.; Liscio, A.; Treossi, E.; Ortolani, L.; Morandi, V.; Pugno, N. M.; Palermo, V., Fragmentation and exfoliation of 2-dimensional materials: a statistical approach. Nanoscale 2014, 6, 5926-5933.

102. De, S.; King, P. J.; Lotya, M.; O'Neill, A.; Doherty, E. M.; Hernandez, Y.; Duesberg, G. S.; Coleman, J. N., Flexible, Transparent, Conducting Films of Randomly Stacked Graphene from Surfactant-Stabilized, Oxide-Free Graphene Dispersions. Small 2010, 6, 458-464.

103. Higgins, T. M.; McAteer, D.; Coelho, J. C. M.; Sanchez, B. M.; Gholamvand, Z.; Moriarty, G.; McEvoy, N.; Berner, N. C.; Duesberg, G. S.; Nicolosi, V.; Coleman, J. N., Effect of Percolation on the Capacitance of Supercapacitor Electrodes Prepared from Composites of Manganese Dioxide Nanoplatelets and Carbon Nanotubes. ACS Nano 2014, 8, 9567-9579. 
104. Gholamvand, Z.; McAteer, D.; Harvey, A.; Backes, C.; Coleman, J. N., Electrochemical Applications of Two-Dimensional Nanosheets: The Effect of Nanosheet Length and Thickness. Chem. Mater. 2016, 28, 2641-2651.

105. McAteer, D.; Gholamvand, Z.; McEvoy, N.; Harvey, A.; O'Malley, E.; Duesberg, G. S.; Coleman, J. N., Thickness Dependence and Percolation Scaling of Hydrogen Production Rate in MoS2 Nanosheet and Nanosheet-Carbon Nanotube Composite Catalytic Electrodes. ACS Nano 2016, $10,672-83$

106. Mendoza-Sanchez, B.; Rasche, B.; Nicolosi, V.; Grant, P. S., Scaleable ultra-thin and high power density graphene electrochemical capacitor electrodes manufactured by aqueous exfoliation and spray deposition. Carbon 2013, 52, 337-346.

107. Dresselhaus, M. S.; Jorio, A.; Saito, R., Characterizing Graphene, Graphite, and Carbon Nanotubes by Raman Spectroscopy. Ann. Rev. Cond. Matter Phys. 2010, 1, 89-108.

108. Cançado, L. G.; Jorio, A.; Ferreira, E. H. M.; Stavale, F.; Achete, C. A.; Capaz, R. B.; Moutinho, M. V. O.; Lombardo, A.; Kulmala, T. S.; Ferrari, A. C., Quantifying Defects in Graphene via Raman Spectroscopy at Different Excitation Energies. Nano Lett. 2011, 11, 3190-3196.

109. Eckmann, A.; Felten, A.; Mishchenko, A.; Britnell, L.; Krupke, R.; Novoselov, K. S.; Casiraghi, C., Probing the Nature of Defects in Graphene by Raman Spectroscopy. Nano Lett. 2012, $12,3925-3930$. 
ToC Figure

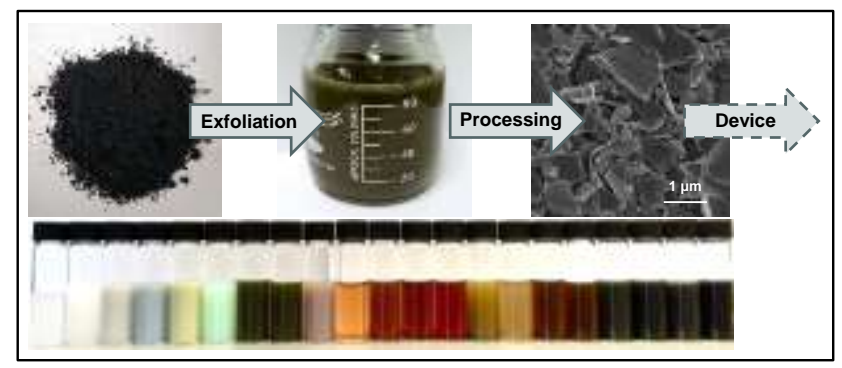

